\title{
PARTICLE SWARM OPTIMISATION AIDED SEMI-BLIND JOINT MAXIMUM LIKELIHOOD CHANNEL ESTIMATION AND DATA DETECTION FOR MIMO SYSTEMS
}

\author{
H.R. Palally, S. Chen, W. Yao and L. Hanzo
}

School of Electronics and Computer Science, University of Southampton, Southampton SO17 1BJ, UK. E-mails: \{hrp1v07, sqc, wy07r, 1h\}@ecs.soton.ac.uk

\begin{abstract}
A novel scheme of semi-blind joint maximum likelihood (ML) channel estimation and data detection is proposed for multipleinput multiple-output (MIMO) systems by decomposing the joint ML optimisation over channel and data into an iterative two-level optimisation loop. Particle swarm optimisation (PSO) is invoked at the upper level to identify the unknown MIMO channel while an enhanced ML sphere detector is used at the lower level to detect the transmitted data. The scheme is semi-blind as a minimum pilot overhead is employed to aid the initialisation of the PSO based channel estimator.
\end{abstract}

Index Terms - Multiple-input multiple-output, joint maximum likelihood estimation, particle swarm optimisation

\section{INTRODUCTION}

MIMO technologies are widely adopted in practice to improve the system capacity and/or quality of service $[1,2]$. Training-based schemes are capable of accurately estimating a MIMO channel at the expense of considerable reduction in system throughput. Blind methods not only impose high complexity and slow convergence but also suffer from unavoidable estimation and decision ambiguities [3]. To overcome this ambiguity problem, a few training symbols are usually employed, and this leads to many semi-blind methods $[4,5$, $6,7,8,9]$. In particular, the work [9] developed a semi-blind scheme of joint ML channel estimation and data detection in which the joint ML optimisation is decomposed into two levels. At the upper level a global search algorithm, known as the repeated weighted boosting search (RWBS) [10], searches for an optimal channel estimate, while at the lower level a ML detector, called the optimised hierarchy reduced search algorithm (OHRSA) detector [11], recovers the transmitted data. Joint ML channel estimation and data detection is achieved by iteratively exchanging information between the RWBS channel estimator and the OHRSA data detector. A minimum number of training symbols are used to provide an initial least squares channel estimate (LSCE) [12] for aiding the RWBS channel estimator to speed up convergence and to avoid the ambiguities inherent in pure blind methods.
In this contribution, we propose a PSO aided semi-blind scheme for joint ML channel estimation and data detection. PSO is a population based stochastic optimisation technique inspired by social behaviour of bird flocking or fish schooling [13]. The PSO method is popular owing to its simplicity in implementation, ability to quickly converge to a reasonably good solution and robustness against local minima. It has been successfully applied to wide-ranging optimisation problems $[14,15,16,17,18]$. Because the PSO method is a very efficient global search algorithm, we achieve considerable complexity saving for the proposed semi-blind joint ML scheme. Specifically, for the same $4 \times 4$ MIMO system, our experimental results show that the PSO aided semi-blind joint scheme requires $20 \%$ less computation than our previous scheme [9]. Throughout our discussions, boldface capitals and lower-case letters stand for matrices and vectors, respectively, while $\mathbf{I}_{K}$ and $\mathbf{1}_{K \times L}$ denote the $K \times K$ identity matrix and the $K \times L$ matrix of unity elements, respectively. $E$ [] denotes the expectation operator, while $\Re[]$ and $\Im[]$ represent the real and imaginary parts, respectively. Finally, $j=\sqrt{-1}$.

\section{MIMO SYSTEM MODEL}

Consider a MIMO system consisting of $n_{T}$ transmitters and $n_{R}$ receivers, which communicates over flat fading channels. The system is described by the well-known MIMO model

$$
\mathbf{y}(k)=\mathbf{H} \mathbf{s}(k)+\mathbf{n}(k),
$$

where $k$ is the symbol index, $\mathbf{H}$ denotes the $n_{R} \times n_{T}$ MIMO channel matrix, $\mathbf{s}(k)=\left[s_{1}(k) s_{2}(k) \cdots s_{n_{T}}(k)\right]^{T}$ is the transmitted symbols vector of the $n_{T}$ transmitters with the symbol energy given by $E\left[\left|s_{m}(k)\right|^{2}\right]=\sigma_{s}^{2}$ for $1 \leq m \leq n_{T}$, $\mathbf{y}(k)=\left[y_{1}(k) y_{2}(k) \cdots y_{n_{R}}(k)\right]^{T}$ denotes the received signal vector, and $\mathbf{n}(k)=\left[n_{1}(k) n_{2}(k) \cdots n_{n_{R}}(k)\right]^{T}$ is the complexvalued Gaussian white noise vector associated with the MIMO channels with $E\left[\mathbf{n}(k) \mathbf{n}^{H}(k)\right]=2 \sigma_{n}^{2} \mathbf{I}_{n_{R}}$.

The modulation scheme is assumed to be quadrature phase shift keying (QPSK). The narrowband MIMO channel matrix is defined by $\mathbf{H}=\left[h_{p, m}\right]$, for $1 \leq p \leq n_{R}$ and $1 \leq$ $m \leq n_{T}$, where $h_{p, m}$ denotes the channel coefficient linking the $m$ th transmitter to the $p$ th receiver. The fading is assumed to be sufficiently slow, so that during the time period 
of a short block of $L$ symbols, all the entries in the MIMO channel matrix $\mathbf{H}$ may be deemed unchanged. From frame to frame, the channel impulse response taps $h_{p, m}$ are independently and identically distributed complex-valued Gaussian processes with zero mean and $E\left[\left|h_{p, m}\right|^{2}\right]=1$. The signalto-noise ratio (SNR) is defined by $E_{b} / N_{o}=\sigma_{s}^{2} / 2 \sigma_{n}^{2}$.

\section{PSO AIDED SEMI-BLIND SCHEME}

Let $\mathbf{Y}=[\mathbf{y}(1) \mathbf{y}(2) \cdots \mathbf{y}(L)]$ be the $n_{R} \times L$ matrix of received data and $\mathbf{S}=[\mathbf{s}(1) \mathbf{s}(2) \cdots \mathbf{s}(L)]$ the $n_{T} \times L$ matrix of transmitted symbols. The probability density function of received data matrix $\mathbf{Y}$ conditioned on MIMO channel matrix $\mathbf{H}$ and transmitted symbol matrix $\mathbf{S}$ can be written as

$$
p(\mathbf{Y} \mid \mathbf{H}, \mathbf{S})=\frac{1}{\left(2 \pi \sigma_{n}^{2}\right)^{n_{R} \times L}} e^{-\frac{1}{2 \sigma_{n}^{2}} \sum_{k=1}^{L}\|\mathbf{y}(k)-\mathbf{H s}(k)\|^{2}} .
$$

The ML estimation of $\mathbf{S}$ and $\mathbf{H}$ can be obtained by jointly maximising $p(\mathbf{Y} \mid \mathbf{H}, \mathbf{S})$ over $\mathbf{S}$ and $\mathbf{H}$. Equivalently, the joint ML estimation is obtained by minimsing the cost function

$$
J_{\mathrm{ML}}(\check{\mathbf{S}}, \check{\mathbf{H}})=\frac{1}{n_{R} \times L} \sum_{k=1}^{L}\|\mathbf{y}(k)-\check{\mathbf{H}} \check{\mathbf{s}}(k)\|^{2} .
$$

Thus the joint ML channel and data estimation is obtained as

$$
(\hat{\mathbf{S}}, \hat{\mathbf{H}})=\arg \left\{\min _{\check{\mathbf{S}}, \check{\mathbf{H}}} J_{\mathrm{ML}}(\check{\mathbf{S}}, \check{\mathbf{H}})\right\} .
$$

The joint ML optimisation defined in (4) is computationally prohibitive. The complexity of this optimisation process may be reduced to a tractable level, if it is decomposed into an iterative search carried out over all the possible data symbols first and then over the channel matrices as

$$
(\hat{\mathbf{S}}, \hat{\mathbf{H}})=\arg \left\{\min _{\breve{\mathbf{H}}}\left[\min _{\breve{\mathbf{S}}} J_{\mathrm{ML}}(\check{\mathbf{S}}, \check{\mathbf{H}})\right]\right\} .
$$

At the inner-level optimisation we use the OHRSA ML detector [11] to find the ML data estimate for the given channel. At the outer-level optimisation we employ a PSO algorithm to search for a global optimal channel estimate. A joint ML solution is achieved with the following iterative loop.

Outer-level Optimisation: The PSO algorithm searches the MIMO channel parameter space via evolving a swarm of channel matrices known as particles to find a global optimal estimate $\hat{\mathbf{H}}$ by minimising the mean square error (MSE)

$$
J_{\mathrm{MSE}}(\check{\mathbf{H}})=J_{\mathrm{ML}}(\hat{\mathbf{S}}(\check{\mathbf{H}}), \check{\mathbf{H}}),
$$

where $\hat{\mathbf{S}}(\check{\mathbf{H}})$ denotes the ML estimate of the transmitted data for the given channel $\mathbf{H}$.

Inner-level Optimisation: Given $\mathbf{H}$ the OHRSA detector finds the ML estimate of the transmitted data and feeds back the ML metric $J_{\mathrm{MSE}}(\check{\mathbf{H}})$ to the upper level.
A few training symbols are employed to provide an initial LSCE for aiding the PSO algorithm. Let the number of training symbols be $K$, and denote the available training data as $\mathbf{Y}_{K}=[\mathbf{y}(1) \mathbf{y}(2) \cdots \mathbf{y}(K)]$ and $\mathbf{S}_{K}=[\mathbf{s}(1) \mathbf{s}(2) \cdots \mathbf{s}(K)]$. The LSCE based on $\left\{\mathbf{Y}_{K}, \mathbf{S}_{K}\right\}$ is readily given by

$$
\check{\mathbf{H}}_{\mathrm{LSCE}}=\mathbf{Y}_{K} \mathbf{S}_{K}^{H}\left(\mathbf{S}_{K} \mathbf{S}_{K}^{H}\right)^{-1} .
$$

To maintain the system throughput, we only use the minimum number of training symbols, namely, $K=n_{T}$. The training symbol matrix $\mathbf{S}_{K}$ is designed to have $n_{T}$ orthogonal rows. This yields the most efficient estimate and removes the need for matrix inversion. With $S$ denoting the swarm size and index $l$ the iteration step, we can now summarise the PSO aided semi-blind joint ML estimation scheme.

A swarm of particles, $\left\{\check{\mathbf{H}}_{i}^{(l)}\right\}_{i=1}^{S}$, that represent potential solutions are initialised as $\check{\mathbf{H}}_{1}^{(0)}=\check{\mathbf{H}}_{\mathrm{LSCE}}$ and

$$
\check{\mathbf{H}}_{i}^{(0)}=\check{\mathbf{H}}_{\mathrm{LSCE}}+\eta\left(\mathbf{1}_{n_{R} \times n_{T}}+j \mathbf{1}_{n_{R} \times n_{T}}\right), 2 \leq i \leq S,
$$

where $\eta$ is the uniformly distributed random variable defined in the range $[-\alpha, \alpha]$. Each particle $\check{\mathbf{H}}_{i}^{(l)}$ has a cost $F\left(\check{\mathbf{H}}_{i}^{(l)}\right)$ associated with it, which is evaluated at each iteration by the OHRSA detector as $F\left(\check{\mathbf{H}}_{i}^{(l)}\right)=J_{\mathrm{MSE}}\left(\check{\mathbf{H}}_{i}^{(l)}\right)$. Each particle $\check{\mathbf{H}}_{i}^{(l)}$ remembers its best position visited so far, denoted as $\mathbf{P} \mathbf{b}_{i}^{(l)}$, which provides the cognitive information. Every particle also knows the best position visited so far among the entire swarm, denoted as $\mathbf{G} \mathbf{b}^{(l)}$, which provides the social information. The cognitive information $\left\{\mathbf{P b}_{i}^{(l)}\right\}_{i=1}^{S}$ and social information $\mathbf{G} \mathbf{b}^{(l)}$ are updated at each iteration

$$
\begin{aligned}
& \text { For }(i=1 ; i \leq S ; i++) \\
& \quad \quad \text { If }\left(F\left(\check{\mathbf{H}}_{i}^{(l)}\right)<F\left(\mathbf{P b}_{i}^{(l)}\right)\right) \quad \mathbf{P b}_{i}^{(l)}=\check{\mathbf{H}}_{i}^{(l)} ; \\
& \text { End for; } \\
& i^{*}=\arg \min _{1 \leq i \leq S} F\left(\mathbf{P} \mathbf{b}_{i}^{(l)}\right) ; \\
& \text { If }\left(F\left(\mathbf{P b}(l)<i^{(l)}\right)<F\left(\mathbf{G b}^{(l)}\right)\right) \quad \mathbf{G b}^{(l)}=\mathbf{P b}_{i^{*}}^{(l)} ;
\end{aligned}
$$

Each particle $\check{\mathbf{H}}_{i}^{(l)}$ also has its velocity, denoted as $\mathbf{V}_{i}^{(l)}$, to direct its flying. In each iteration, the velocity and the position of the $i$ th particle are updated according to

$$
\begin{aligned}
\mathbf{V}_{i}^{(l+1)}= & w * \mathbf{V}_{i}^{(l)}+\operatorname{rand}() * c_{1} *\left(\mathbf{P} \mathbf{b}_{i}^{(l)}-\check{\mathbf{H}}_{i}^{(l)}\right) \\
& +\operatorname{rand}() * c_{2} *\left(\mathbf{G} \mathbf{b}^{(l)}-\check{\mathbf{H}}_{i}^{(l)}\right), \\
\check{\mathbf{H}}_{i}^{(l+1)}= & \check{\mathbf{H}}_{i}^{(l)}+\mathbf{V}_{i}^{(l+1)},
\end{aligned}
$$

where $w$ is the inertia weight, $\operatorname{rand}()$ denotes the uniform random number between 0 and 1 , and $c_{1}$ and $c_{2}$ are the two acceleration coefficients. In order to avoid excessive roaming of particles beyond the search space [16], a velocity range $\left[-V_{\max }, V_{\max }\right]$ is imposed on each element of $\mathbf{V}_{i}^{(l+1)}$

$$
\begin{aligned}
& \text { If }\left(\Re\left[\left.\mathbf{V}_{i}^{(l+1)}\right|_{p, q}\right]>V_{\max }\right) \quad \Re\left[\left.\mathbf{V}_{i}^{(l+1)}\right|_{p, q}\right]=V_{\text {max }} ; \\
& \text { If }\left(\Re\left[\left.\mathbf{V}_{i}^{(l+1)}\right|_{p, q}\right]<-V_{\max }\right) \quad \Re\left[\left.\mathbf{V}_{i}^{(l+1)}\right|_{p, q}\right]=-V_{\text {max }} ; \\
& \text { If }\left(\Im\left[\left.\mathbf{V}_{i}^{(l+1)}\right|_{p, q}\right]>V_{\max }\right) \quad \Im\left[\left.\mathbf{V}_{i}^{(l+1)}\right|_{p, q}\right]=V_{\max } ; \\
& \text { If }\left(\Im\left[\left.\mathbf{V}_{i}^{(l+1)}\right|_{p, q}\right]<-V_{\max }\right) \quad \Im\left[\left.\mathbf{V}_{i}^{(l+1)}\right|_{p, q}\right]=-V_{\max } ;
\end{aligned}
$$


where $\left.\mathbf{V}\right|_{p, q}$ denotes the $(p, q)$ th element of $\mathbf{V}$. Moreover, if the velocity (9) approaches zero, it is reinitialised to proportional to $V_{\max }$ with a small factor $\gamma$

$$
\left.\mathbf{V}_{i}^{(l+1)}\right|_{p, q}= \pm \operatorname{rand}() * \gamma *\left(V_{\max }+j V_{\max }\right) .
$$

Our empirical results suggest that $\gamma=0.1$ is appropriate. Similarly, each element of $\check{\mathbf{H}}_{i}^{(l+1)}$ is checked to ensure that it stays inside the search space $\left[-H_{\max }, H_{\max }\right]$

$$
\begin{aligned}
& \text { If }\left(\Re\left[\left.\check{\mathbf{H}}_{i}^{(l+1)}\right|_{p, q}\right]>H_{\max }\right) \quad \Re\left[\left.\check{\mathbf{H}}_{i}^{(l+1)}\right|_{p, q}\right]=H_{\max } ; \\
& \text { If }\left(\Re\left[\left.\check{\mathbf{H}}_{i}^{(l+1)}\right|_{p, q}\right]<-H_{\max }\right) \quad \Re\left[\left.\check{\mathbf{H}}_{i}^{(l+1)}\right|_{p, q}\right]=-H_{\max } \text {; } \\
& \text { If }\left(\Im\left[\left.\check{\mathbf{H}}_{i}^{(l+1)}\right|_{p, q}\right]>H_{\max }\right) \quad \Im\left[\left.\check{\mathbf{H}}_{i}^{(l+1)}\right|_{p, q}\right]=H_{\max } \text {; } \\
& \text { If }\left(\Im\left[\left.\check{\mathbf{H}}_{i}^{(l+1)}\right|_{p, q}\right]<-H_{\max }\right) \quad \Im\left[\left.\check{\mathbf{H}}_{i}^{(l+1)}\right|_{p, q}\right]=-H_{\max } ;
\end{aligned}
$$

Let $C_{\text {OHRSA }}(L)$ be the complexity of the OHRSA algorithm to decode the $L$-symbol data matrix $\mathbf{S}$ and let $N_{\text {OHRSA }}$ be the number of calls for the OHRSA algorithm required by the PSO algorithm to converge. Then the complexity of the proposed semi-blind method is expressed as

$$
C=N_{\text {OHRSA }} \times C_{\text {OHRSA }}(L),
$$

where $C_{\text {OHRSA }}(L)$ is given in [11], and $N_{\text {OHRSA }}=S \times I_{\max }$ with $I_{\max }$ denoting the maximum number of iterations

We adopt a time varying acceleration coefficient (TVAC) mechanism in which $c_{1}$ varies from 0.5 to 2.5 and $c_{2}$ changes from 2.5 to 0.5 during the iterative procedure according to

$$
\begin{aligned}
& c_{1}=(2.5-0.5) \frac{l}{I_{\max }}+0.5, \\
& c_{2}=(0.5-2.5) \frac{l}{I_{\max }}+2.5 .
\end{aligned}
$$

This TVAC is different from the one suggested in [15] and it works well for our application. We also remove the influence of the previous velocity by setting $w=0$, as suggested

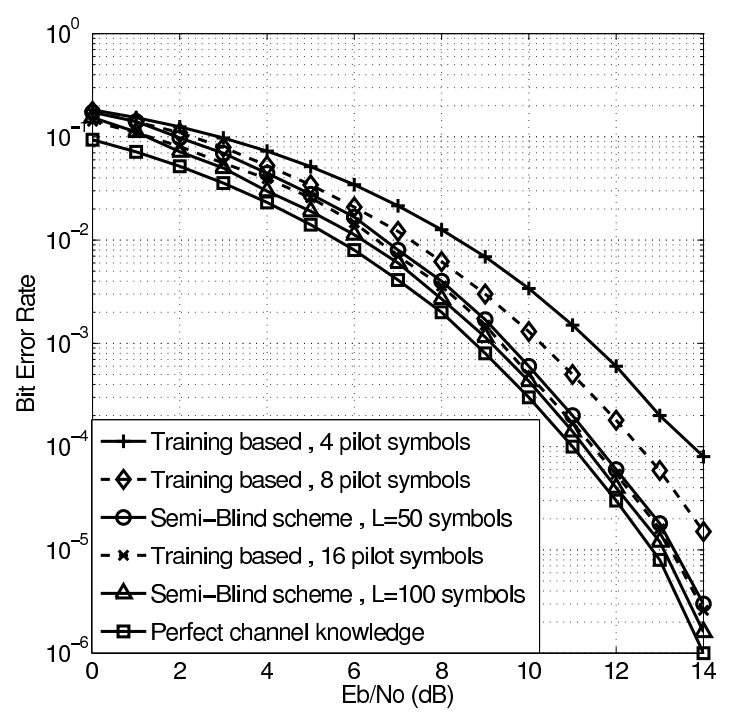

Fig. 1. Bit error rate of the PSO aided semi-blind scheme with two different values of frame length $L$ after 1000 OHRSA evaluations, in comparison with the training-based cases using 4,8 and 16 pilot symbols as well as the case of perfect channel knowledge. in [15]. We set the population size to $S=20$ and the maximum number of evolutionary steps to $I_{\max }=50$, which appear adequate for our application. The control parameter $\alpha$ in the channel population initialisation is found empirically to be $\alpha=0.15$. Since $\Re\left[h_{p, q}\right]$ and $\Im\left[h_{p, q}\right]$ of each MIMO channel tap $h_{p, q}$ are Gaussian distributed with a variance 0.5 , we choose the search space limit to $H_{\max }=1.8$ which lies between 2 to 3 standard deviations of the true tap distribution. We also set the velocity limit to $V_{\max }=1.0$ which is confirmed in simulation to be a suitable value for our application.

\section{SIMULATION STUDY}

A simulation study was carried out to investigate the PSO aided semi-blind joint ML channel estimation and data detection scheme. We considered a MIMO system with $n_{T}=4$ and $n_{R}=4$. The achievable performance was assessed in the simulation using three metrics, and these were the MSE defined in (6), the mean channel error (MCE) defined as

$$
J_{\mathrm{MCE}}(\check{\mathbf{H}})=\|\mathbf{H}-\check{\mathbf{H}}\|^{2},
$$

where $\mathbf{H}$ denotes the true MIMO channel matrix and $\check{\mathbf{H}}$ the channel estimate, and the bit error ratio (BER). As we set $S=20$ and $I_{\max }=50$, the complexity of the PSO based semi-blind scheme was determined by $N_{\text {OHRSA }}=1000$.

Fig. 1 depicted the BER performance of the PSO based semi-blind scheme having two frame lengths $L=50$ and 100 after 1000 OHRSA evaluations, in comparison with the performance of the training-based OHRSA detector having $K=$ 4,8 and 16 training symbols, respectively, as well as with the case of perfect channel knowledge. The results shown in Fig. 1 were averaged over 50 different channel realisations. It can be observed from Fig. 1 that, for the training-based

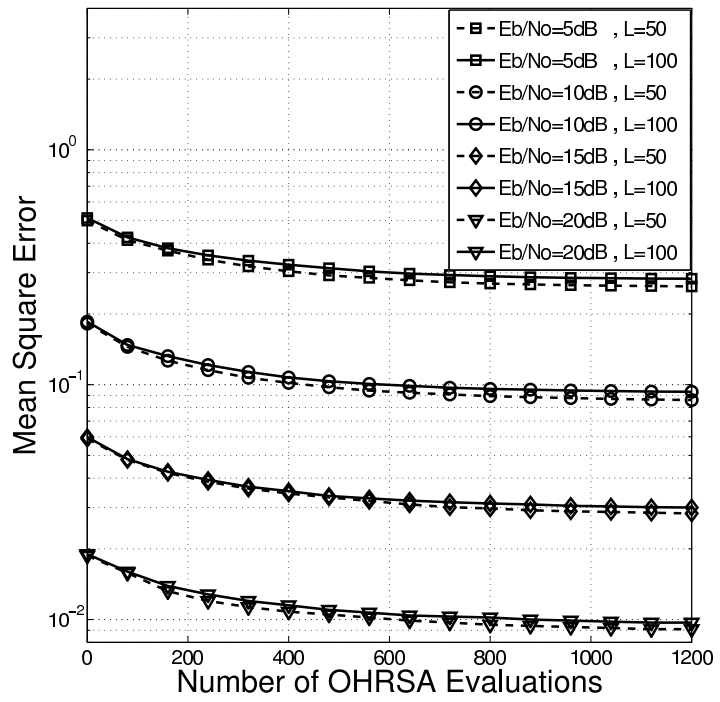

Fig. 2. Convergence of mean square error averaged over 50 different channel realisations for different values of $E_{b} / N_{o}$ and $L$. 


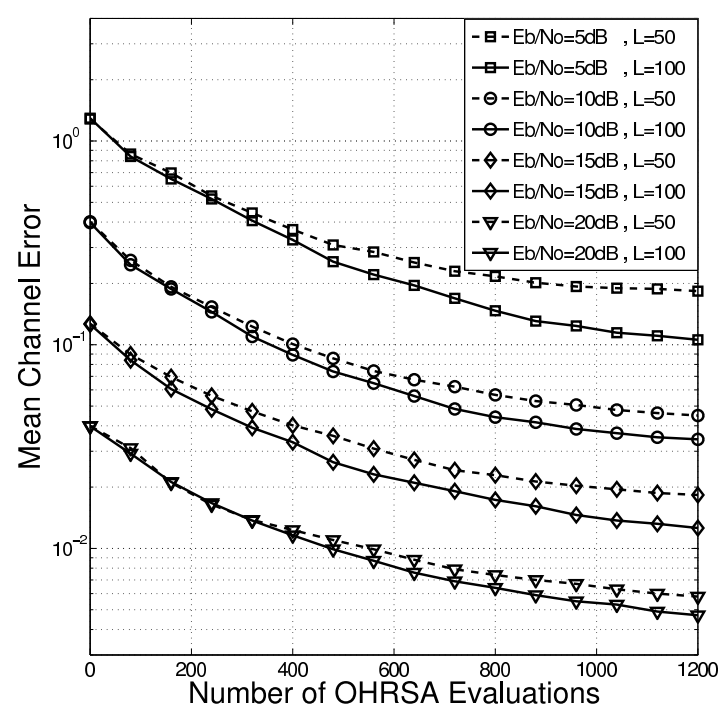

Fig. 3. Convergence of mean channel error averaged over 50 different channel realisations for different values of $E_{b} / N_{o}$ and $N$.

scheme to achieve the same BER performance of the semiblind one having a frame length $L=100$ and with only 4 pilot symbols, the number of training symbols had to be more than 16. This example was identical to the MIMO system investigated in [9]. The BER performance of the PSO-based semi-blind scheme depicted in Fig. 1 was slightly better than the BER of the RWBS-based semi-blind scheme shown in [9]. Moreover, the performance of the PSO-based scheme was achieved after 1000 OHRSA evaluations, while the performance of the RWBS-based scheme was obtained after 1200 OHRSA evaluations. Thus, the proposed PSO-based semiblind method achieved $20 \%$ saving in computation.

Figs. 2 and 3 depict the convergence performance of the proposed PSO-aided semi-blind scheme averaged over 50 different channel realisations in terms of the MSE and MCE, respectively, for different SNR values as well as for two frame lengths $L=50$ and 100. It can be seen from Fig. 2 that the MSE converged to the noise floor. The MCE performance shown in Fig. 3 was seen to be slightly better and converging faster than the result shown in [9].

\section{REFERENCES}

[1] T. L. Marzetta and B. M. Hochwal, "Capacity of a mobile multiple-antenna communication link in Rayleigh flat fading," IEEE Trans. Information Theory, vol. 45, pp. 139-157, January 1999.

[2] A. J. Paulraj, D. A. Gore, R. U. Nabar and H. Bölcskei, “An overview of MIMO communications - A key to gigabit wireless," Proc. IEEE, vol. 92, pp. 198-218, February 2004.

[3] L. Tang, R. W. Liu, V. C. Soon and Y. F. Huang, "Indeterminacy and identifiability of blind identification," IEEE Trans. Circuits and Systems, vol. 38, pp. 499-509, May 1991.
[4] A. Medles and D. T. M. Slock, "Semiblind channel estimation for MIMO spatial multiplexing systems," in Proc. VTC2001Fall, Atlantic City, USA, Oct.7-11, 2001, vol. 2, pp. 12401244.

[5] C. Cozzo and B. L. Hughes, "Joint channel estimation and data detection in space-time communications," IEEE Trans. Communications, vol. 51, pp. 1266-1270, August 2003.

[6] S. Buzzi, M. Lops and S. Sardellitti, "Performance of iterative data detection and channel estimation for single-antenna and multiple-antennas wireless communications," IEEE Trans. Vehicular Technology, vol. 53, pp. 1085-1104, July 2004.

[7] T. Wo, P. A. Hoeher, A. Scherb and K. D. Kammeyer, "Performance analysis of maximum-likelihood semiblind estimation of MIMO channels," in Proc. VTC2006-Spring, Melbourne, Australia, May 7-10, 2006, vol. 4, pp. 1738-1742.

[8] A. K. Jagannatham and B. D. Rao, "Whitening-rotation-based semi-blind MIMO channel estimation," IEEE Trans. Signal Processing, vol. 54, pp. 861-869, March 2006.

[9] M. Abuthinien, S. Chen and L. Hanzo, "Semi-blind joint maximum likelihood channel estimation and data detection for MIMO systems," IEEE Signal Processing Letters, vol. 15, pp. 202-205, 2008.

[10] S. Chen, X. X. Wang and C.J. Harris, "Experiments with repeating weighted boosting search for optimization in signal processing applications," IEEE Trans. System, Man and Cybernetics, Part B, vol. 35, pp. 682-693, August 2005.

[11] J. Akhtman, A. Wolfgang, S. Chen and L. Hanzo, "An optimized-hierarchy-aided approximate Log-MAP detector for MIMO systems," IEEE Trans. Wireless Communications, vol. 6, pp. 1900-1909, May 2007.

[12] M. Biguesh and A. B. Gershman, "Training-based MIMO channel estimation: A study of estimator tradeoffs and optimal training signals," IEEE Trans. Signal Processing, vol. 54, pp. 88-893, March 2006.

[13] J. Kennedy and R. Eberhart, "Particle swarm optimization," in Proc. of 1995 IEEE Int. Conf. Neural Networks, Perth, Australia, Nov. 27 - Dec. 1, 1995, vol. 4, pp. 1942-1948.

[14] J. Kennedy and R. C. Eberhart, Swarm Intelligence, Morgan Kaufmann, 2001.

[15] A. Ratnaweera, S. K. Halgamuge and H. C. Watson, "Selforganizing hierarchical particle swarm optimizer with timevarying acceleration coefficients," IEEE Trans. Evolutionary Computation, vol. 8, pp. 240-255, June 2004.

[16] S. M. Guru, S. K. Halgamuge and S. Fernando, "Particle swarm optimisers for cluster formation in wireless sensor networks," in Proc. 2005 Int. Conf. Intelligent Sensors, Sensor Networks and Information Processing, Melbourne, Australia, Dec. 5-8, 2005, pp. 319-324.

[17] H.-M. Feng, "Self-generation RBFNs using evolutional PSO learning," Neurocomputing, vol. 70, pp. 241-251, December 2006.

[18] K. K. Soo, Y. M. Siu, W. S. Chan, L. Yang and R. S. Chen, "Particle-swarm-optimization-based multiuser detector for CDMA communications," IEEE Trans. Vehicular Technology, vol. 56, pp. 3006-3013, September 2007. 